\title{
A Comparative Study of Medical Dramas in China, South Korea, and the United States
}

\author{
Jiachen Li \\ School of Literatures, Laguages \& Cultures, University of Edinburgh, UK
}

\begin{abstract}
The medical drama first appeared in the 1960s and since then it has become one of the most popular television genres, and always gets a relatively high score and audience rating. Many scholars have conducted extensive research on the subject, but mostly, such research has been limited to American medical dramas. Thus, this study conducted a comparative analysis to examine how the image of the physician and physician-patient relationships are represented in American, Korean and Chinese medical dramas. It aims to establish which form the dominant physician-patient relationship models within each national context. By implementing a content analysis on The Good Doctor, Dr. Romantic and The Surgeon, this paper has found that American medical dramas represent more positive characteristics of physicians than Korean and Chinese medical dramas. Chinese and Korean medical dramas pay more attention to representing the negative characteristics of physicians, especially the "corrupt" characteristic. Besides, medical disputes are a common social problem in both Chinese and Korean medical dramas, and different physician-patient relationship models depend on different circumstances.
\end{abstract}

Keywords: Medical drama, Physician-patient relationship, Representation, Cultural differences.

\section{Introduction}

Because of the huge cultural and social differences between countries, people in different countries have different perceptions of medical treatment and the physician-patient relationship. For instance, The Farewell (2019) tells the story about the grandmother of a Chinese family who has cancer, and the family members decided not to tell her the truth because they thought that telling her the truth would make her afraid, which is not good for her health or helps her treatment. However, the granddaughter, who grew up in the United States, cannot completely understand it. Owing to the impact of Confucian family ethics of China, family members are involved in medical decision making to a considerable extent and they usually play an important role in physician-patient communication. Sometimes patients do not even know their own health condition, because knowing the truth depends on whether their families are willing to tell them. This indicates that Chinese and west countries have very different views on medical treatment and family concepts. Thus, this paper intends to investigate the different representations of this medical drama in different countries.

Although watching TV dramas is generally regarded as an indispensable way of entertainment, it also involves the function of providing information and education. Asbeek Brusse et al. (2015) called this unique educational function of entertainment contexts "entertainment-education" (p. 396). Such functions are common in medical dramas since TV dramas serve as a major source of health information for most audiences. Additionally, the educational significance of medical dramas is also widely applied to the teaching of medical students, especially for the teaching of humanities. The vivid scenes in medical drama can help students understand patients' grief, fragility and fear so as to foster students' empathy and compassion.

However, in order to establish and maintain the richness and attraction of the storyline, medical dramas always develop a complicated and romantic love story or fierce professional rivalry, often violating the ethical and professional norms of the vast majority of physicians (Goodman, 2007, p. 183).
Even though the audience has practical knowledge of relative issues, TV drama still remained a strong influence (Kato et al., 2017 , p. 10). This implies that medical drama constantly plays a vital role in the construction of an audience's expectation of physicians and physician-patient relationships. The representation of a physician's image is continuously changing, from the perfect hero image of the 1960s to a negative depiction in the 1980s. To the present day, the image of physicians has become more diversified and controversial and particularly, when centred on the physician-patient relationship (Virzi et al., 2011, p. 275). Based on the different socio-cultural backgrounds and medical system, different countries represent physicians' image and physician-patient relationships differently. Therefore, this study will conduct a comparative study on the representation of the physician's image and physician-patient relationships by using well-known and recent medical drama of America, China and Korea.

\section{Literature Review}

\subsection{Previous Studies on the Content of Medical Drama}

With the apparent transnational spread and popularity, a large number of scholars have carried out extensive research on medical drama. First, illness treatment is central to medical drama, and there has been wide-ranging previous research on how medical drama represents different types of diseases, such as cardiovascular diseases, cancer, injury,etc..Among those researched, ER and Grey's Anatomy are the most common research cases(Van De Blunt and Damian,2004; Ye and Ward 2010). However, the gravity of chronic illnesses such as diabetes, hypertension and coronary heart disease are underestimated, virtually (Chung, 2014). Moreover, the cure rate of using cardiopulmonary resuscitation on television is twice as high as in reality, which could be regarded as both a miracle and misinformation (Diem et al., 1996). Furthermore, the mortality rate of patients who check into the emergency room in medical dramas is almost nine times higher than in the real world. Even though it is necessary to increase the dramatic quality, it can provoke fear as well, particularly among people without hospitalisation experience (Hetsroni, 
2009, p. 311). Nevertheless, the exaggerated depiction on TV could also bring benefits to the audience. Eisenman et al. (2015) found that even though the cure rate of CPR would always be overestimated in medical dramas, it could, in fact, help audiences learn the CPR skill and save cardiac arrest victims in real life.

\subsection{Previous Studies on the Portrayal of Physician and Patient}

As the main character of medical dramas, the portrayal of physicians has become the focus of scholars' study, especially their gender, age, race and personality. Physicians have always been depicted as a perfect, messianic, hero figures in early medical dramas, especially before the 1990s (Chory-Assad and Tamborini, 2001). Since the 1990s, the images of physicians in medical dramas have changed considerably, in particular, the negative images of physicians have increased. Chory-Assad and Tamborini (2001) explained that such change is caused by medical consumerism in which the physician-patient encounter is regarded as a commercial transaction (p. 500). On the one hand, the character became more complicated and realistic. On the other hand, such portrayals will allow audiences to witness the negative aspect of physicians' personalities, which may reduce their respect and worship for physicians, affect patient-physician interactions and even undermine their confidence in physicians. Chory-Assad and Tamborini (2001) made a content analysis, which showed that although in terms of competence, physical attractiveness, power, wealth and status, physicians were still depicted positively overall, they were deficient in personality, especially in the dimension of interpersonal style (p. 503). Jain and Slater (2013) studied the gender and race of the representation of physicians in medical dramas. The results showed that the number of male and female physicians portrayed in the medical dramas was largely consistent with the proportion in the real world. However, there is a major gap between racial representation in medical drama and reality.

\subsection{Portrayal and Models of Physician-patient Relationship}

Tian and Yoo (2020) claimed that watching medical dramas is positively associated with the trust of doctors, and trust could mediate clinical outcomes. Before the 1990s. physicians always played a leading role in the physician-patient relationship, while, their patients were rarely given a voice (Hetsroni, 2009). Such physician-patient relationship is referred to as the paternalistic model. However, such unequal physician-patient relationship does not meet patients' expectations. According to the investigation of Vick and Scott (1998), patients expected to gain more information and they believed that the most important issue was "being able to talk to the doctor" (p. 587). Besides, Quill and Brody (1996) asserted that in order to conform to patients' autonomy, "guided paternalism" is the best service model.

Virzi et al. (2011) divided physician-patient relationships into three models. these were Activity-Passivity (the physician has the right to make decisions, representing as the active subject, while, the patient becomes the passive object of his diagnosis and therapeutic choices); Guide-Cooperation (physicians guide patients to make medical decisions through effective communication); Mutual Comprehension (the relationship between physicians and patients is assumed to be as equal as possible, and both sides try to understand their differences in terms of knowledge, needs, expectations and fears) (p. 277). The result showed that $61.3 \%$ of the representations of physician-patient relationship were based on the Activity-Passivity model, $35.6 \%$ representations belonged to Guide-Cooperation model, whereas the Mutual Comprehension model hardly exists.

\section{Research Gap and Question}

In the present review, there are three main findings: 1) Watching medical dramas has a profound impact on the audience's health- care attitude and behaviour, meanwhile, medical drama is also an important educational tool for medical students. Thus, it is of great importance to study medical dramas. 2) The image of the physician and physician-patient relationships are constantly developing, with previous studies on the physician's image and physician-patient relationship being normally based on $E R$, Grey's Anatomy, House M.D. and Chicago Hope. Thus, it is necessary to research new medical dramas. 3) Most studies of medical drama are limited to American medical dramas. Few scholars have conducted systematical research on Asian medical dramas. Therefore, this paper will take the latest and well-known medical dramas in the US, South Korea and China as the research objects, to comparatively analyse the representations of physicians and physician-patient relationships under the different socio-cultural backgrounds.

The research questions are proposed as below:

RQ1. How is the image of the physician and physician-patient relationships represented in American, Korean and Chinese medical dramas?

RQ2. Which form the dominant models within each national context?

\section{Theoretical Framework\& Methodology}

Framing theory has been widely used as a theoretical guide in a variety of social science fields. Framing theory refers to "the principles of selection, emphasis and presentation composed of little tacit theories about what exists, what happens, and what matters" (Gitlin, 2003, p. 6). It indicates that framing theory is commonly used in media representation studies to analyse how mass media represents specific content to the audience through selecting and highlighting. Since this paper aims to analyse the representation of physician image and physician-patient relationship, framing theory, especially its sociological conception, will be utilised as a theoretical guide.

Additionally, Content analysis is a widely used analysis approach in qualitative research. Previous studies indicated that content analysis could directly show the research results of the content of medical dramas through clear data. More importantly, it could ascertain the content that the media attempts to emphasise or ignore. Therefore, in this study, content analysis will be applied as the main method to analyse 
a physician's image and physician-patient relationships of medical dramas under the guidance of framing theory.

\subsection{Sample}

Generally, this study reviewed 136 episodes of popular medical dramas from the past five years to compare the specific content of medical dramas of China, America and South Korea. In order to ensure the similar sample length (of time) of each country, this study selected Surgeons (2017), Romantic (2016), Dr. Romantic 2 (2020) and The Good Doctor (2017-2019) as research samples. Surgeons once broadcasted on Beijing satellite TV and Zhejiang satellite TV and achieved high audience rating. At the same time, it broadcasts on Tencent Video, IQIYI video and LETV streaming media platforms with a great amount of total broadcast up to 7.8 billion times. Dr. Romantic (2016) was the viewer rating champion among the TV dramas broadcast at that period. Meanwhile, Dr. Romantic 2 is the first Korean drama to surpass 20\% TV viewer rating in 2020 (Jang, 2020). The Good Doctor is a popular American medical drama television series which won numerous awards. The average viewership of the first, the second and the third seasons are 15.61 million, 12.2 million and 10.82 million, respectively. All the episodes of these medical dramas could be acquired through DVD or streaming. Besides, to ensure the reliability of the research data of the content analysis method, this paper applied intercoder reliability testing. The primary author as the first coder is in charge of researching all the samples. While the second coder takes charge of Surgeons (2017). Comparing the percentage of the same data implemented, the deviation is trifling and acceptable.

\subsection{Coding of Physician's Image}

Basically, the image of a physician could be divided into two frames: the positive frame and the negative frame. To be specific, in the positively framed storyline, physicians are framed as competent, empathetic, stable, benevolent, humorous and responsible. On the contrary, in the negatively framed storyline, physicians are framed as incompetent, corrupt, rude, prejudiced and irresponsible. To code accurately, each trait will be counted through the embodiment of specific behaviours.

"Competent"- The success of the operation or first aid treatment will be regarded as direct proof of the doctor's professional performance. Besides, the dialogues involving a large number of medical terminologies can also be regarded as the embodiment of professional competence. Hence, when the drama representing professional discussions about disease, or representing a successful treatment, it will be counted as one time of competent frame.

"Empathetic"- According to Deloney and Graham (2003), empathy and compassion play important roles in the physician-patient relationship. Physicians will express their emotional care to their patients by giving utterances such as "I understand how you feel", "I know it's hard", "I know you're suffering" and "You will be fine". Meanwhile, nonverbally empathy representations will be coded through the behaviours such as holding the patient's hand, supportively patting their shoulder, hugging them, showing sadness or even weeping for their patients.

"Stable"- When an emergency occurs (someone suddenly faints or is injured), the physician can always keep calm and deal with it quickly while others are panicking. Even more, physicians usually encounter some unexpected conditions during the operation, "typically characterised by uncontrolled blood splattering amidst the quickening pace of warning beeps from electronic indicators" (Khiun, 2011, p. 255). Hence, it will be coded as stable if the physician keeps calm and takes crucial action promptly.

"Benevolent"- The characteristic of benevolence could be embodied by charitable behaviour, like making a donation. Or other ways to help patients solve the problem of operating costs, such as organising charity events, helping patients seek help from charitable foundations, government agencies or insurance companies.

"Humorous"- Although physicians need to keep calm and steady in the operating theatre, humour plays a vital role in patient-centred care during daily consultation (Scholl, 2007). A physician's sense of humour can be reflected in the conversations with patients or colleagues. It will be coded if the physician amuses patients or colleagues.

"Responsible"- It will be coded as responsible if the physician sacrificed private time (such as sleep time, eating time and holidays) to perform emergency surgery, or saying "It's on me" when they face the risk of surgery.

"Incompetent"- The surgical failure, to a great extent, represents the incompetence of the physician from the audience's viewpoint, even though it might be due to a variety of reasons. While, medical malpractice is completely a physicians' fault, and can often happen to interns or young physicians who are inexperienced in the face of emergencies.

"Corrupt"- Bribery, corruption and money politics are prevalent in medical dramas. The wealthy patients want to fast track their operations by bribing the physicians. A few corrupt physicians even take special care of the wealthy patients initiatively to get their help in the future. Therefore, illicit competition, bribery and corruption could be coded as corrupt frame.

"Rude"- Rude behaviour such as fighting, intense and gruff conversation between physicians themselves, and between physicians and their patients will be coded as negative frame.

"Biased"- Bias is manifested in many aspects, including racism, sexism, discriminating people with physical or mental disorders. Such bias could be either towards patients o colleagues.

"Irresponsible"- It will be coded if physicians do not care about their patients, ignore patient's requirements, and handle personal affairs during working hours.

\subsection{Coding of Physician-patient Relationship Model}


Based on the review of the previous articles on the physician-patient relationship models, this paper summarises the common physician-patient relationship models into three categories: Paternalistic Model, Guiding Model and Information Model. In order to code accurately, it will only be coded when the patient needs major surgery.

"Paternalistic model"- In this model, the physician is portrayed in a powerful and authoritative role, diagnosing the illness and making the therapeutic schemes, while the patient is completely submissive. It usually occurs in the emergency room, the patient calls for an immediate operation, so that physicians do not have the time or opportunity to communicate with them. In addition, physicians insist on their own diagnosis and treatment plan and probably claim that "you must...", "you should listen to me". On occasion, physicians even disregard a patient's decision and adhere to their own operating plan.

"Guiding model"- physicians help patients to elucidate values and suggesting what medical interventions realise these values. It will be coded if physicians persuade patients to follow their operative treatment.

"Information model"- The physician only provides the patient with relevant information but is not involved in decision making.

\section{Results}

\subsection{Characteristics of the Major Physician Roles}

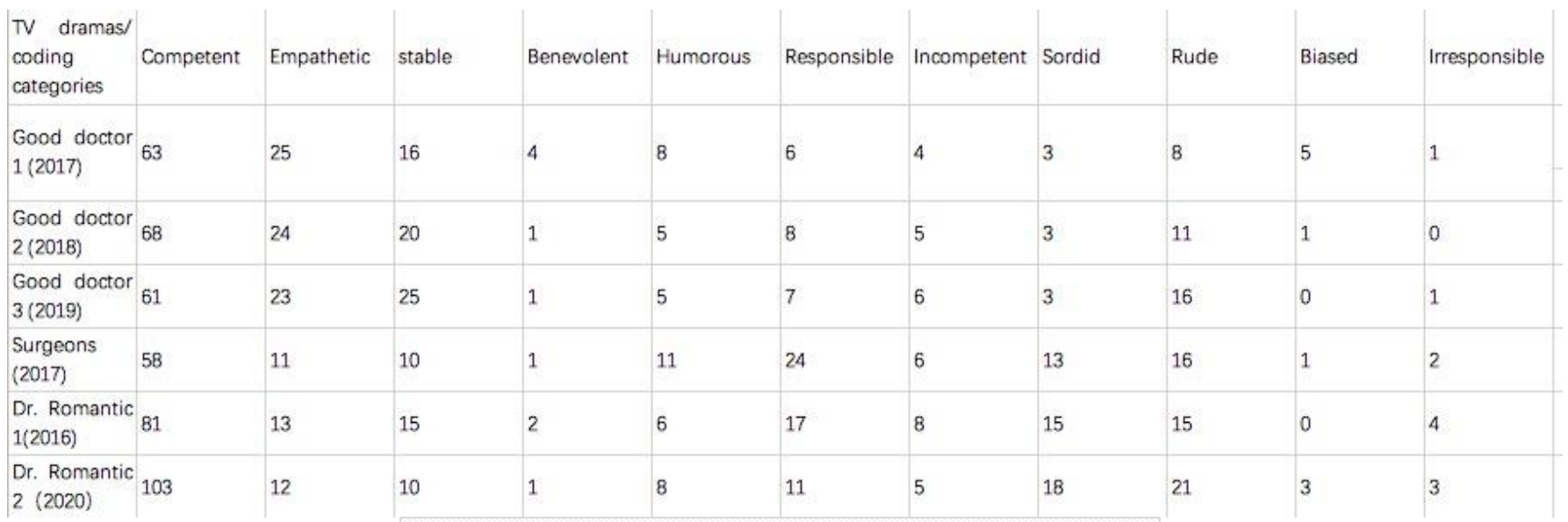

"Competent" is the most emphatic quality of physicians in The Good Doctor, where a large number of professional terms and surgical scenes reflect the professionalism and authenticity of the medical drama. There are various rare cases in The Good Doctor. On the one hand, it shows the competence of physicians, on the other hand, it caters to the audience's imagination of spectacular medical drama. "Empathetic" is the second most frequently represented positive characteristic in The Good Doctor. In the first episode, Dr Marcus Andrews opposed hiring Dr Shaun Murphy, because he thought people who were autistic could communicate effectively with others. He claimed that "a surgeon needs to communicate not just information, but sympathy, and empathy". The representations of the "stable" characteristics of physicians have appeared 61 times in the three seasons of The Good Doctor. Normally, "stable" representations were coded when doctors handled emergency situations during the operation. To some extent, the "stable" characteristic of physicians is to create a tense atmosphere and enhance the dramatic effect to stimulate the audience.

Compared with the positive frames, the proportion of negative characteristics representations is relatively small, accounting for $15 \%$ in total. Physicians are rarely portrayed as incompetent $(3 \%)$. In most instances, the deaths of patients are due to the difficulty and risk of surgery. The characteristic of "rude" accounts for half of the negative frames, however, such a negative characteristic is not the drawback of the majority of physicians. In most cases, Dr Shaun Murphy is the initiator of the quarrel. Because of autism, Shaun sometimes cannot control his emotions. As the protagonist, Shaun's change and growth is an important part of the drama.

The representation of "biased" only accounts for $1 \%$. It was coded when another physician thinks Shaun cannot be a good doctor because of autism. It is noticeable, that although female physicians once complained about the difficulties and injustices they face in the workplace, gender and racial discrimination is almost non-existent in The Good Doctor. In The Good Doctor, there are 9 main physician characters, three are female, accounting for $33.3 \%$ of the total, which is slightly higher than the actual ratio. In terms of the physicians' race, The Good Doctor has paid more attention to racial diversity. And both white and non-white physicians have demonstrated excellent professional competence. 


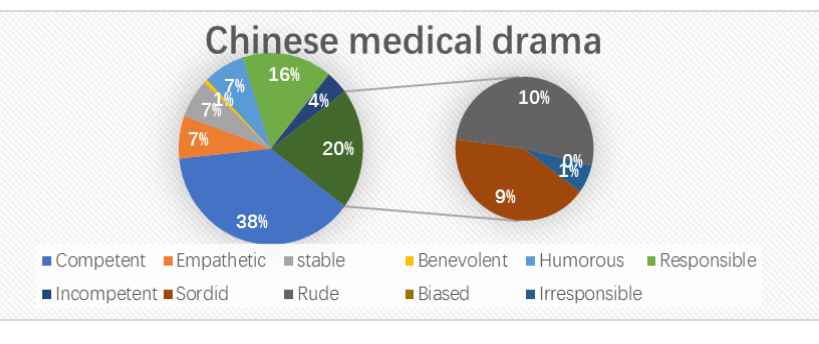

Although the "competent" frame accounts for the largest proportion of physicians' characteristics, professional terms and surgical scenes have not been shown frequently (58 times in total). Compared with The Good Doctor, the plot development of Surgeon has moved slowly. The description of one patient's condition can last for three or four episodes. Furthermore, the mainline of the plot is the protagonist's investigation of his mother's medical malpractice and it is interspersed with the emotional development of the hero and heroine, so that Surgeon as a medical drama is not sufficiently professional. "Responsible" is the second most frequently represented positive characteristic of physicians in Surgeon. On the one hand, a responsible characteristic reflects the hard work of doctors. On the other hand, responsible refers to the situation that physicians are willing to take great risks to treat patients, even if they know they will face difficult surgery and the accusations of a patient's family members. Actually, the traditional moral value and problematic legal system make Chinese physicians shoulder extra responsibilities. Even though there is no medical malpractice, if the patient is not cured, the family members probably will blame the physician. When the patient's family member sues the physician, court decisions are usually in favour of the patients' families. According to Chen (2019), "doctors were asked to make compromises to the best of their abilities to stop patients' family members from disturbing the orderly medical services" (p. 13). Nevertheless, Surgeon emphasises the "responsible" characteristic of physicians, which means that even in the face of family members' accusations and lawsuits, physicians still try their best to save the lives of patients.

The proportions of other positive frames such as empathetic, stable, humorous are similar, at about $7 \%$. While, negative frames account for $24 \%$ in total, "rude" and "corrupt" frames account for a large percentage, $10 \%$ and $9 \%$ respectively. Misunderstanding between physicians and patients can lead to arguments and even physical conflict. "Corrupt" is the second most frequently represented negative characteristic, behaviours such as illicit competition, bribery and corruption have occurred many times in Surgeon. Surgeon reflects the collusion between the physician and the pharmaceutical sales representative. Many physicians voluntarily recommend the drug of Pioneer Medical Company to their patients. Compared with other companies' drugs, this chemotherapeutic drug is a little more expensive, thus, physicians could get a larger kickback. On the one hand, it exposed the greed of certain physicians. On the other hand, as Dr Yang Fan claimed in the drama, it revealed the real problem that the salaries of Chinese physicians do not recompense their hard work.

"Biased" characteristic of physicians has not been represented in Surgeon. Physicians are fair to their patients and colleagues without discrimination. However, this medical drama embodies the prejudice of patients. For instance, there is a patient who questioned Dr Lu's abilities because she looks young and is female. Besides, there are gender stereotypes in the portrayal of female physicians. Firstly, there are only two female physicians (Dr. Lu and Dr. Chu) in Surgeon, with other main roles of physicians all played by men. Although Dr Lu has strong professional abilities and is more competent than many male physicians, her position was replaced by the male leading role, Dr Zhuang Shu, who is more capable than her. Because of her impulse and sensibility, Dr Zhuang helped her settle disputes with patients and the chief physicianon several occasions. Also, among all the interns, Dr Chu is the worst and she has made a few foolish mistakes. To some extent, Surgeon overstates female physician's shortcomings and implies the vulnerability of females.

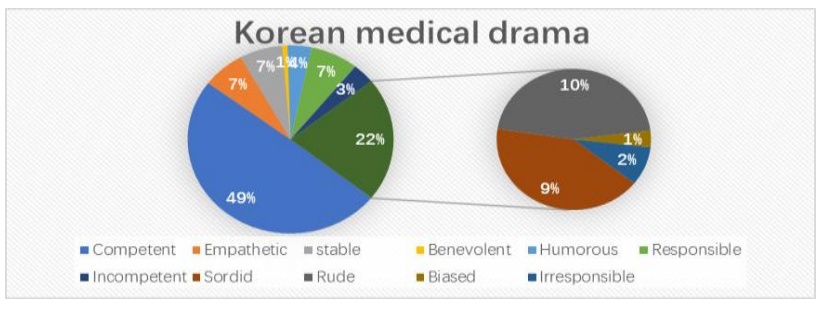

The proportion of "competent" characteristics of physicians in Korean medical dramas is the highest among the three countries. The dialogue between physicians in Dr. Romantic involves a large number of medical terms. Also, to show the professionalism of medical drama, it gives a detailed explanation of the terms in the form of subtitles. The proportion of other positive frames of Korean medical drama are similar to Chinese medical drama. "Empathetic" and "stable" characteristics both account for 7\%. While, "humour" and "responsible" characteristics account for $4 \%$ and $7 \%$, respectively. The proportion of negative frames $(25 \%)$ is also consistent with the proportion in Chinese medical drama (24\%). It was significant that the proportions of "rude" characteristics $(10 \%)$ and "corrupt" characteristics $(9 \%)$ are the same as in Chinese medical drama.

"Corrupt" has been coded 33 times in Dr. Romantic, accounting for $9 \%$ of physician characteristics. Corruption and money politics are the prevalent realism themes, and Korean dramas are keen to reflect various social problems. Dr Romantic reflects the serious problem of military violence and the belief that "the preservation of the bureaucratic order and stability of the medical fraternity should come before the integrity and professionalism of medical practice" (Khiun, 2011 , p. 257) "Biased" frame was coded three times in $D r$. Romantic, which shows gender discrimination of male physicians to female physicians. In most Asian medical dramas, the roles of superiors and senior physicians are played by men. As Khiun (2011) stated, "males are not only shown to naturally possess medical expertise but also as having moral and bureaucratic authority" (p. 255).

\subsection{Physician-patient relationship model}

Physician-Patient Relationship Model

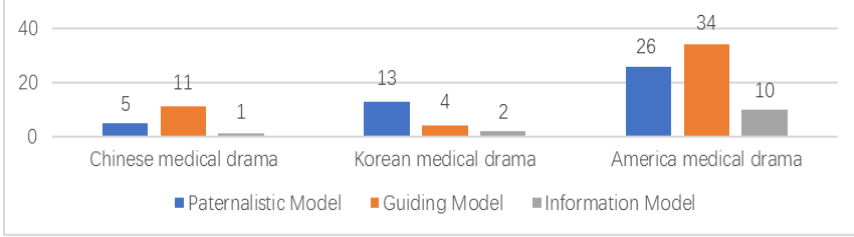


In both Chinese and American medical dramas, the Guiding Model is the one that is represented most frequently. Generally, physicians will guide patients to make a decision, especially when patients refuse surgical therapy, but physicians believe that surgery is necessary, they will try their best to persuade the patient to receive treatments. For instance, in the seventeenth episode of Surgeon, a single mother was worried that she would not be able to afford to raise a seriously ill child in the future, so she decided to give up the treatment. To encourage this patient, Dr Zhuang contacted the parent of a former patient who had successfully been cured after the surgery. The healthy and lovely boy gave her hope, and finally she agreed to sign the operation consent.

Paternalistic Model also accounts for a large proportion in medical dramas. For instance, in The Good Doctor, a girl was circumcised (female genital mutilation) when she was a child, so that she came to the hospital for vaginal rejuvenation. Dr Lim told them there are two options: one is clitoral reconstruction and the other is ablating the peripheral nerves and remove the remaining clitoral tissue, which is safer. Under the torture of pain and the expectation of her parents, she chose to take it out. But Dr Lim felt her hesitation and misery. Thus, she still did the surgery of clitoral reconstruction with the risk of revoking her medical license. She ignored the patient's own decision and chose that she believed was best for the patient. Even though such behaviour is illegal, it could be understood and would be supported by the audience from the humanitarian and moral dimension.

In Korean medical drama, the Paternalistic Model is the dominant physician-patient relationship model. On the one hand, in Dr. Romantic, many patients are in a state of unconsciousness, they need physicians to make decisions. On the other hand, Korean medical drama shows the high authority of physicians. According to Goodyear-Smith and Buetow (2001), "the medical profession has been viewed as a typical example of a patriarchal system: the doctor, usually male with high social status, wealth and exclusive knowledge, making decisions on behalf of all patients, women in particular" (p. 450). In Dr. Romantic, Dr Kim has excellent medical skills and absolute authority in the hospital. Therefore, he was used to asking patients to listen to his opinions or making quick decisions for patients when they were unconscious.

In the medical dramas of the three countries, the information model is the least common. According to Chin (2002), "if in the name of honouring autonomy and freedom, physicians merely offer possible options without any professional input, then this informative model is unlikely to serve patient's interests" (p. 153). Thus, information model is not common in daily life. In the sample of medical dramas studied in this paper, only when physicians cannot judge which choice is more beneficial to their patient, will they give the right's decision to the patients themselves.

\section{Discussion}

Compared with Korean and Chinese medical dramas, American medical dramas represent more positive characteristics of physicians. American medical dramas tend to demonstrate the "competent" characteristic of physicians through performing operations on complex and rare cases, and it also stress the "empathetic" and "stable" characteristics of physicians. It indicates that the physician is the perfect combination of sensibility and rationality and shows the physician's care for patients. While, in Korean and Chinese medical drams, the positive characteristics of physicians account for three-quarters in total. For Korean medical drama, each proportion of the positive characteristic of physicians is similar to others, except for the "competent" characteristic. A large number of terminology dialogues is applied to show the professionalism of physicians, which also improves the authenticity and credibility of medical drama. While, in Chinese medical drama, the professionalism of is relatively poor, physicians' moral sense and the characteristic of responsibility are magnified. Because of the unique social environment, it seems that Chinese physicians are under greater pressure and have to shoulder extra responsibilities.

To increase dramatic conflict, draw the audience's attention and keep tension, "rude" frame is the most common characteristics in all the three countries' medical dramas, which is always represented through opposing, quarrelling or arguing. In addition, Chinese and Korean medical dramas focus on representing "corrupt" characteristic of physicians. Specifically, corrupt characteristic accounts for $9 \%$ of physicians' characteristic frames in both Chinese and Korean medical dramas. According to Chen (2019), "the struggle between pursuing medical professionalism and maintaining bureaucratic order has always been the focus of Asian medical dramas" (p. 4). In Asian medical dramas, except for having excellent professional ability, good physicians have to struggle to overcome bureaucratic hurdles, which means that they must also abide by high moral standards. By constructing the negative image of some physicians, especially by representing corrupt characteristic, Chinese medical drama reflects the defects of the medical system and exposes the collusion between power and money in medical institutions. Likewise, Korean medical dramas also reflect the power struggle and unfair career competition in medical institutions. Nevertheless, there are relatively few negative portrayals of physicians in American medical dramas. Corrupt behaviours such as buying doughnuts for superiors or flattering superiors verbally could even be ignored. Besides, by representing the "biased" characteristic, American medical dramas reflect the difficulties that women face in the workplace. But meanwhile, it gives full affirmation to women's competence. However, in Korean and Chinese medical dramas, gender stereotypes still exist.

In terms of physician-patient relationship model, the research result of Chinese medical drama and American medical drama are similar and reasonable. The guiding model accounts for the largest share of the three models, followed by the paternalistic model. This is because patients might make irrational decisions when they are in a state of emotional complexity. At that time, physicians' responsibilities and obligations should not be simplified. They should not only serve as information providers but should guide patients to make the most beneficial decisions. Additionally, due to the huge gap of professional knowledge between physicians and patients, physicians are normally in a dominant position, the paternalistic model is also represented frequently in medical dramas. For Korean medical drama, the paternalistic model is 
the most common. Asian medical dramas seem to emphasise the power of physicians, especially the power and authority of male physicians. However, it does not mean that patients' rights have been ignored or simply shows the oppression of patriarchal society. Under certain circumstances, the physician's independent decision seems to be the most effective and time-saving way. Overall, each of the three types of therapeutic relationship is appropriate for some specific cases, and none of them should be denied.

\section{Limitation}

Because the research only focuses on the content of the medical dramas, there is a lack of empirical investigation of physicians' characteristic and physician-patient relationship in real life as well as the audience's attitudes towards the image of physicians. Moreover, there is a lack of knowledge of the real effect on the audience's perception of physicians' image. Therefore, further research is required to focus on the physicians' characteristics in real life and physicians-patients relationship as well as the audience's perception. This can be achieved by implementing different research methods such as observation, experiments or surveys.

\section{References}

[1] Asbeek Brusse, E. D., Fransen, M. L., \& Smit, E. G. (2015). Educational storylines in entertainment television: audience reactions toward persuasive strategies in medical dramas. Journal of health communication, 20(4), 396-405.

[2] Chen, L. (2019). Vulnerable live patients, powerful dead patients: A textual analysis of doctor-patient relationships in popular Chinese medical dramas. Cogent Arts \& Humanities, 6(1), 1622626.

[3] Chin, J. J. (2002). Doctor-patient relationship: from medical paternalism to enhanced autonomy. Singapore medical journal, 43(3), 152-155.

[4] Chory-Assad, R. M., \& Tamborini, R. (2001). Television doctors: An analysis of physicians in fictional and non-fictional television programs. Journal of Broadcasting \& Electronic Media, 45(3), 499-521.

[5] Chung, J. E. (2014). Medical dramas and viewer perception of health: testing cultivation effects. Human Communication Research, 40(3), 333-349.

[6] Deloney, L. A., \& Graham, C. J. (2003). Developments: Wit: Using drama to teach first-year medical students about empathy and compassion. Teaching and learning in medicine, 15(4), 247-251.

[7] Diem, S. J., Lantos, J. D., \& Tulsky, J. A. (1996). Cardiopulmonary resuscitation on television: Miracles and misinformation: New Engl J Med 1996; 334/24: 1578-1582. Resuscitation, 33(1), 96.

[8] Eisenman, A., Rusetski, V., Zohar, Z., Avital, D., \& Stolero, J. (2015). Subconscious passive learning of CPR techniques through television medical drama. Australasian Journal of Paramedicine, 3(3).

[9] Gitlin, T. (2003). The whole world is watching: Mass media in the making and unmaking of the new left. Univ of California Press.

[10] Goodman, K. (2007). Imagining doctors: Medical students and the TV medical drama. AMA Journal of Ethics, 9(1), 182-187.
[11] Goodyear-Smith, F., \& Buetow, S. (2001). Power issues in the doctor-patient relationship. Health care analysis, 9(4), 449-462.

[12] Hetsroni, A. (2009). If you must be hospitalized, television is not the place: Diagnoses, survival rates and demographic characteristics of patients in TV hospital dramas. Communication Research Reports, 26(4), 311-322.

[13] Jain, P., \& Slater, M. D. (2013). Provider portrayals and patient-provider communication in drama and reality medical entertainment television shows. Journal of health communication, 18(6), 703-722.

[14] Jang (2020). "Dr. Romantic 2" becomes first drama to surpass $20 \%$ TV rating in 2020. Retrieved from https://www.kdramapal.com/dr-romantic-2-becomes-fir st-drama-surpass-20-tv-rating-2020/.

[15] Kato, M., Ishikawa, H., Okuhara, T., Okada, M., \& Kiuchi, T. (2017). Mapping research on health topics presented in prime-time TV dramas in "developed" countries: a literature review. Cogent Social Sciences, 3(1), 1318477.

[16] Khiun, L. K. (2011). Post-Confucian East Asian television dramas: staging medical politics inside the White Tower. International Journal of Cultural Studies, 14(3), 251-264.

[17] Quill, T. E., \& Brody, H. (1996). Physician recommendations and patient autonomy: finding a balance between physician power and patient choice.

[18] Scholl, J. C. (2007). The use of humor to promote patient-centered care. Journal of Applied Communication Research, 35(2), 156-176.

[19] Tian, Y., \& Yoo, J. H. (2020). Medical drama viewing and medical trust: A moderated mediation approach. Health Communication, 35(1), 46-55.

[20] Van den Bulck, J., \& Damiaans, K. (2004). Cardiopulmonary resuscitation on Flemish television: Challenges to the television effects hypothesis. Emergency Medicine Journal, 21, 565-567.

[21] Vick, S., \& Scott, A. (1998). Agency in health care. Examining patients' preferences for attributes of the doctor-patient relationship. Journal of health economics, 17(5), 587-605.

[22] Virzi, A., Dipasquale, S., Signorelli, M., Aguglia, E., Bianchini, O., \& Palermo, F. (2011). Movie portrayals of physicians and the doctor-patient relationship. Journal of Cognitive and Behavioral Psychotherapies, 6(2), 475-485.

[23] Ye, Y., \& Ward, K. E. (2010). The depiction of illness and related matters in two top-ranked primetime network medical dramas in the United States: a content analysis. Journal of Health Communication, 15(5), 555-570. 